\title{
An arithmetic group associated with a Pisot unit, and its symbolic-dynamical representation
}

\author{
by \\ Nikita Sidorov (Manchester)
}

1. The definition of the Pisot group and its basic properties. Let $\beta>1$ be a Pisot number, i.e. an algebraic integer whose conjugates have the moduli strictly less than 1 . Let the characteristic polynomial of $\beta$ be $g(x)=x^{m}-k_{1} x^{m-1}-k_{2} x^{m-2}-\ldots-k_{m}$. We assume $\beta$ to be a unit, i.e. $k_{m}= \pm 1$.

We recall that since $\beta$ is a Pisot number, $\left\|\beta^{n}\right\| \rightarrow 0$ as $n \rightarrow+\infty$, where $\|y\|=\min \{|y-k|: k \in \mathbb{Z}\}$. Let

$$
\mathcal{P}_{\beta}=\left\{\xi:\left\|\xi \beta^{n}\right\| \rightarrow 0, n \rightarrow+\infty\right\} .
$$

Let us first establish some auxiliary facts. It is well known that $\mathcal{P}_{\beta} \subset \mathbb{Q}(\beta)$ (see, e.g., [Cas]). Let $\operatorname{Tr}(\xi)$ denote the trace of $\xi$, i.e. the sum of $\xi$ and all its conjugates.

Lemma 1.1. The set $\mathcal{P}_{\beta}$ is a commutative group under addition containing $\mathbb{Z}[\beta]$. It can be characterized as follows:

$$
\mathcal{P}_{\beta}=\{\xi \in \mathbb{Q}(\beta): \operatorname{Tr}(a \xi) \in \mathbb{Z} \text { for any } a \in \mathbb{Z}[\beta]\} .
$$

Proof. The first claim is a consequence of the inequalities $\left\|\xi_{1} \pm \xi_{2}\right\| \leq$ $\left\|\xi_{1}\right\|+\left\|\xi_{2}\right\|$ and of the fact that $\left\|\beta^{n}\right\| \rightarrow 0$. To prove (1.1), we observe that the classical theorem due to Pisot and Vijayaraghavan says that for any Pisot $\beta$, there exists $k_{0} \in \mathbb{N}$ such that $\mathcal{P}_{\beta}=\left\{\xi \in \mathbb{Q}(\beta): \operatorname{Tr}\left(\beta^{k} \xi\right) \in \mathbb{Z}, k \geq k_{0}\right\}$ (see, e.g., [Cas]). Hence (1.1) follows, because $\beta$ is a unit, whence $\beta^{-1}$ is an algebraic integer.

Thus, if we regard $\mathbb{Z}[\beta]$ as a lattice over $\mathbb{Z}$, then by (1.1) and the definition, $\mathcal{P}_{\beta}$ is the dual lattice for $\mathbb{Z}[\beta]$ (notation: $\mathcal{P}_{\beta}=(\mathbb{Z}[\beta])^{*}$ ). The following claim follows from some general statement about lattices [FrolTa, Chapter III, (2.20)].

2000 Mathematics Subject Classification: 11R06, 11R04, 37B10, 37D20.

Supported by the EPSRC grant ref. no. GRL98923. 
Proposition 1.2. There exists $\xi_{0} \in \mathbb{Q}(\beta) \backslash \mathbb{Z}[\beta]$ such that

$$
\mathcal{P}_{\beta}=\xi_{0} \cdot \mathbb{Z}[\beta] .
$$

It can be given by the formula $\xi_{0}=\left(g^{\prime}(\beta)\right)^{-1}$.

Definition. Let the Pisot group $\mathcal{A}_{\beta}$ be defined as the quotient group $\mathcal{P}_{\beta} / \mathbb{Z}[\beta]$.

Let $\beta_{1}=\beta$, and $\beta_{2}, \beta_{3}, \ldots, \beta_{m}$ denote its conjugates. Finally, let $D=$ $D(\beta)=\prod_{i \neq j}\left(\beta_{i}-\beta_{j}\right)^{2}$ be the discriminant of $\beta$.

Theorem 1.3. The order of the Pisot group is $|D(\beta)|$.

Proof. Again, we will use some facts from classical number theory. Let

$$
M_{\beta}=\left(\begin{array}{cccc}
\operatorname{Tr}(1) & \operatorname{Tr}(\beta) & \ldots & \operatorname{Tr}\left(\beta^{m-1}\right) \\
\operatorname{Tr}(\beta) & \operatorname{Tr}\left(\beta^{2}\right) & \ldots & \operatorname{Tr}\left(\beta^{m}\right) \\
\ldots & \ldots & \ldots & \ldots \\
\operatorname{Tr}\left(\beta^{m-1}\right) & \operatorname{Tr}\left(\beta^{m}\right) & \ldots & \operatorname{Tr}\left(\beta^{2 m-1}\right)
\end{array}\right)
$$

For any lattice $M$ over $\mathbb{Z}$,

$$
M^{*}: M \cong \mathbb{Z}^{m} / M_{\beta} \mathbb{Z}^{m},
$$

whence the order of $M^{*}: M$ is $|D|$, where $D=\operatorname{det} M_{\beta}$ (see [FrolTa, Chapter III]).

It suffices to show that $\operatorname{det} M_{\beta}=D(\beta)$. Sometimes, this relation is taken as the definition of the discriminant. Observe that $M_{\beta}=V_{\beta} \cdot V_{\beta}^{T}$, where

$$
V_{\beta}=\left(\begin{array}{cccc}
1 & 1 & \ldots & 1 \\
\beta_{1} & \beta_{2} & \ldots & \beta_{m} \\
\beta_{1}^{2} & \beta_{2}^{2} & \ldots & \beta_{m}^{2} \\
\ldots & \ldots & \ldots & \cdots \\
\beta_{1}^{m-1} & \beta_{2}^{m-1} & \ldots & \beta_{m}^{m-1}
\end{array}\right),
$$

whence $\operatorname{det} M_{\beta}=\left(\operatorname{det} V_{\beta}\right)^{2}=\prod_{i \neq j}\left(\beta_{i}-\beta_{j}\right)^{2}=D$.

The following simple fact answers the question about the finer structure of the Pisot group. Let $d=\min \left\{l \geq 1: l M_{\beta}^{-1} \in \mathbb{Z}^{m \times m}\right\}$.

Lemma 1.4. The Pisot group $\mathcal{A}_{\beta}$ contains $\mathbb{Z} / d \mathbb{Z}$ as a subgroup. In particular, if $d=|D|$, then $\mathcal{A}_{\beta}$ is cyclic, and if $|D| / d$ is prime, then $\mathcal{A}_{\beta}$ is isomorphic to $(\mathbb{Z} / d \mathbb{Z}) \times(\mathbb{Z} /(|D| / d) \mathbb{Z})$.

Proof. This is a direct consequence of (1.2).

Corollary 1.5. Assume the entries of $D M_{\beta}^{-1}$ to be coprime. Then $\mathcal{A}_{\beta}$ is cyclic.

REMARK. All the above results are valid for any algebraic unit if one takes (1.1) for the definition of $\mathcal{P}_{\beta}$. 
EXAMPLES. 1. The quadratic units. Let $\beta^{2}=k \beta \pm 1$ with $k \geq 1$ for +1 and $k \geq 3$ for -1 . Then by direct inspection,

$$
M_{\beta}=\left(\begin{array}{cc}
2 & k \\
k & k^{2} \pm 2
\end{array}\right),
$$

and $\xi_{0}=1 / \sqrt{D}$. Then by Lemma $1.4, \mathcal{A}_{\beta}$ is isomorphic to $\mathbb{Z} / D \mathbb{Z}$ if $k$ is odd, and to $(\mathbb{Z} /(D / 2) \mathbb{Z}) \times(\mathbb{Z} / 2 \mathbb{Z})$ otherwise. The Pisot group for this case was considered in [SV2].

2. Let $\beta$ be the "tribonacci number", i.e. the positive root of $x^{3}=x^{2}+$ $x+1$. Here $D=-44$,

$$
M_{\beta}=\left(\begin{array}{ccc}
3 & 1 & 3 \\
1 & 3 & 7 \\
3 & 7 & 11
\end{array}\right), \quad M_{\beta}^{-1}=\frac{1}{22}\left(\begin{array}{ccc}
8 & -5 & 1 \\
-5 & -12 & 9 \\
1 & 9 & -4
\end{array}\right),
$$

$\xi_{0}^{-1}=g^{\prime}(\beta)=-1-2 \beta+3 \beta^{2}$, and $\xi_{0}=\frac{1}{22}\left(1+9 \beta-4 \beta^{2}\right)$. By Lemma 1.4, $\mathcal{A}_{\beta} \cong(\mathbb{Z} / 22 \mathbb{Z}) \times(\mathbb{Z} / 2 \mathbb{Z})$.

3. Finally, let $\beta$ be the smallest Pisot number, i.e. the root of $\beta^{3}=\beta+1$ (see, e.g., [DuPi]). Here $D=-23$,

$$
M_{\beta}=\left(\begin{array}{lll}
3 & 0 & 2 \\
0 & 2 & 3 \\
2 & 3 & 2
\end{array}\right), \quad M_{\beta}^{-1}=\frac{1}{23}\left(\begin{array}{ccc}
5 & -6 & 4 \\
-6 & -2 & 9 \\
4 & 9 & -6
\end{array}\right),
$$

and since 23 is a prime, $\mathcal{A}_{\beta} \cong \mathbb{Z} / 23 \mathbb{Z}$.

To end this section, we establish a link between the groups $\mathcal{P}_{\beta}$ and $\mathcal{A}_{\beta}$ and certain groups of recurrent sequences, which will be used in the next section. Let

$$
R_{\beta}=\left\{\left\{T_{n}\right\}_{n=1}^{\infty} \in \mathbb{Z}^{\infty} \mid \exists j: T_{n+m}=\sum_{i=1}^{m} k_{i} T_{n+m-i}, n \geq j\right\} .
$$

Obviously, $R_{\beta}$ is a group under addition. Define $h: R_{\beta} \rightarrow \mathcal{P}_{\beta}$ by

$$
h\left(\left\{T_{n}\right\}\right):=\lim _{n \rightarrow+\infty} \beta^{-n} T_{n} .
$$

The image does belong to $\mathcal{P}_{\beta}$, as $\left\|\xi \beta^{n}\right\|=\left|\xi \beta^{n}-T_{n}\right| \rightarrow 0$ (in view of $\beta$ being a Pisot number, whence $\left.\left|\xi-\beta^{-n} T_{n}\right|=o\left(\beta^{-n}\right)\right)$.

Lemma 1.6. The mapping $h$ is an epimorphism of groups.

Proof. It is obvious from the definition that $h$ is a homomorphism. Let $\xi \in \mathcal{P}_{\beta}$. We define $T_{n}$ as the integer closest to $\xi \beta^{n}$. Since $\beta$ is a Pisot number, $\left\{T_{n}\right\}$ will eventually satisfy the corresponding recurrence relation, whence $h^{-1}(\xi) \neq \emptyset$.

Let now the equivalence relation $\sim$ on $R_{\beta}$ be defined as follows: $\left\{T_{n}\right\} \sim$ $\left\{T_{n}^{\prime}\right\}$ iff $\lim _{n} \beta^{-n}\left(T_{n}-T_{n}^{\prime}\right) \in \mathbb{Z}[\beta]$. Then obviously, the quotient map $h^{\prime}$ is an epimorphism as well. 


\section{Symbolic realization of the Pisot group}

Definition ([Pa]). A representation of an $x \in[0,1)$ of the form

$$
x=\psi_{\beta}\left(\varepsilon_{1}, \varepsilon_{2}, \ldots\right):=\sum_{k=1}^{\infty} \varepsilon_{k} \beta^{-k}
$$

is called the $\beta$-expansion of $x$ if the "digits" $\left\{\varepsilon_{k}\right\}_{k=1}^{\infty}$ are obtained by means of the greedy algorithm (similarly to the decimal expansion), i.e., $\varepsilon_{1}=$ $\varepsilon_{1}(x)=[\beta x], \varepsilon_{2}=\varepsilon_{2}(x)=[\beta\{\beta x\}]$, etc. The set of all possible sequences $\left\{\left\{\varepsilon_{k}(x)\right\}_{k=1}^{\infty}: x \in[0,1)\right\}$ is called the (one-sided) $\beta$-compactum and will be denoted by $X_{\beta}^{+}$.

The $\beta$-compactum can be described more explicitly. Let $1=\sum_{k=1}^{\infty} d_{k} \beta^{-k}$ be the expansion of 1 defined by the greedy algorithm, i.e., $d_{1}^{\prime}=[\beta], d_{2}^{\prime}=$ $[\beta\{\beta\}]$, etc. If the sequence $\left\{d_{n}^{\prime}\right\}$ is not finite, we put $d_{n} \equiv d_{n}^{\prime}$. Otherwise let $k=\max \left\{j: d_{j}^{\prime}>0\right\}$, and $\left(d_{1}, d_{2}, \ldots\right):=\left(\overline{d_{1}^{\prime}, \ldots, d_{k-1}^{\prime}, d_{k}^{\prime}-1}\right)$, where the bar denotes a period.

We will write $\left\{x_{n}\right\}_{n=1}^{\infty} \prec\left\{y_{n}\right\}_{n=1}^{\infty}$ if $x_{n}<y_{n}$ for the smallest $n \geq 1$ such that $x_{n} \neq y_{n}$. Then

$$
X_{\beta}^{+}=\left\{\left\{\varepsilon_{n}\right\}_{n=1}^{\infty}:\left(\varepsilon_{n}, \varepsilon_{n+1}, \ldots\right) \prec\left(d_{1}, d_{2}, \ldots\right) \text { for all } n \in \mathbb{N}\right\}
$$

(see $[\mathrm{Pa}]$ ). Similarly, we define the two-sided $\beta$-compactum as

$$
X_{\beta}=\left\{\left\{\varepsilon_{n}\right\}_{n=-\infty}^{\infty}:\left(\varepsilon_{n}, \varepsilon_{n+1}, \ldots\right) \prec\left(d_{1}, d_{2}, \ldots\right) \text { for all } n \in \mathbb{Z}\right\} .
$$

It is shown in $[\mathrm{Pa}]$ that $\psi_{\beta}: X_{\beta}^{+} \rightarrow[0,1)$ is one-to-one everywhere with the exception of some countable set of points.

Both compacta are naturally endowed with the weak topology, i.e. with the topology of coordinatewise convergence. For $\beta$ Pisot the properties of the $\beta$-compactum are well studied. Its main property is that it is sofic, i.e., the shift on $X_{\beta}$ is a factor of a subshift of finite type (see, e.g., the review $[\mathrm{Bl}])$. Let $\operatorname{Fin}(\beta)$ denote the set of $x$ 's whose $\beta$-expansions are finite, i.e. have the tail $0^{\infty}$. Obviously, $\operatorname{Fin}(\beta) \subset \mathbb{Z}[\beta] \cap[0,1)$, but the inverse inclusion does not hold for an arbitrary Pisot unit.

Definition. A Pisot unit is called finitary if

$$
\operatorname{Fin}(\beta)=\mathbb{Z}[\beta] \cap[0,1) .
$$

EXAMPLES. 1. The Pisot number which is the dominant root of the equation $x^{m}=k_{1} x^{m-1}+\ldots+k_{m}, k_{1} \geq k_{2} \geq \ldots \geq k_{m} \geq 1$, is known to be finitary [FrSo].

2. A quadratic Pisot unit is finitary if and only if its norm is +1 . Indeed, if $\beta^{2}=k \beta+1, k \geq 1$, then the claim follows from the previous one. If $\beta^{2}=k \beta-1, k \geq 3$, then in view of $(\beta-1)^{2}=\beta^{2}-2 \beta+1=(k-2) \beta$, 
we have the $\beta$-expansion $\beta-1=(k-2) \beta^{-1}+(k-2) \beta^{-2}+\ldots$, whence $\mathbb{Z}[\beta] \cap[0,1) \not \subset \operatorname{Fin}(\beta)$.

3. For the cubic Pisot units there also exists a full criterion due to S. Akiyama [Ak1]. Namely, the norm of a finitary cubic $\beta$ must be -1 , i.e. $\beta^{3}=k_{1} \beta^{2}+k_{2} \beta+1$. Furthermore, such a $\beta$ is finitary if and only if $k_{1} \geq 0$ and $-1 \leq k_{2} \leq k_{1}+1$. Thus, the tribonacci number and the smallest Pisot number are both finitary, whereas, for example, the principal root of $x^{3}=3 x^{2}-2 x+1$ is not.

4. Finally, the second Pisot number, i.e. the positive root of $x^{4}=x^{3}+1$, is nonfinitary, as $\beta^{-2}+\beta^{-3}=\beta^{-1}+\beta^{-6}+\beta^{-11}+\ldots$

From now on we will assume $\beta$ to be finitary.

REMARK. The $\beta$-expansions can be easily extended from $[0,1)$ to $\mathbb{R}_{+}$in the way analogous to the decimal expansions. This yields the possibility of adding in $X_{\beta}$ two sequences finite to the left. Namely, if $\bar{\varepsilon}$ and $\bar{\varepsilon}^{\prime}$ are both finite to the left, put $x:=\sum_{k=-\infty}^{\infty}\left(\varepsilon_{k}+\varepsilon_{k}^{\prime}\right) \beta^{-k}$; then by definition, $\bar{\varepsilon}+\bar{\varepsilon}^{\prime}$ is the $\beta$-expansion of $x$. The same is true for subtraction, though $\bar{\varepsilon}-\bar{\varepsilon}^{\prime}$ is well defined only for those sequences for which $\sum_{k}\left(\varepsilon_{k}-\varepsilon_{k}^{\prime}\right) \beta^{-k} \geq 0$.

Theorem (A. Bertrand, K. Schmidt). For a Pisot $\beta$, any $x \in \mathbb{Q}(\beta) \cap \mathbb{R}_{+}$ has an ultimately periodic $\beta$-expansion.

Thus, all the elements of $\mathcal{P}_{\beta} \cap \mathbb{R}_{+}$have ultimately periodic $\beta$-expansions.

LEMma 2.1. For the nonnegative elements of $\mathcal{P}_{\beta}$ the set of their periods is finite.

Proof. Let $q=|D|$; then all the denominators of the elements in question in the standard basis of $\mathbb{Q}(\beta)$ are bounded by $q$ (see Proposition 1.2). Therefore, it suffices to show that the intersection of the set of numbers in $(0,1)$ having purely periodic $\beta$-expansions and $\frac{1}{q} \mathbb{Z}[\beta]$ is finite.

The proof is basically the same as that of Lemma 2 of [Ak2]. Namely, it was shown there that for each purely periodic element in $(0,1)$ the values of its conjugates are uniformly bounded. Therefore, after the natural embedding of $\mathbb{Q}(\beta)$ into $\mathbb{R}^{m}$ we will have a lattice lying in a ball, and it will hence be finite.

Our goal is to find a realization of the Pisot group $\mathcal{A}_{\beta}$ in $X_{\beta}$. We will perform the following operation: to a nonnegative $\xi \in \mathcal{P}_{\beta}$ the mapping $\widetilde{g}$ assigns the purely periodic two-sided sequence in $X_{\beta}$ which is the extension to the left of the periodic tail of the $\beta$-expansion of $\xi$. Now let $g$ be the corresponding quotient map from $\mathcal{A}_{\beta}$. To any equivalence class $[\xi] \in \mathcal{A}_{\beta}$ it assigns the class of all purely periodic sequences in $X_{\beta}$ whose tails can be obtained from the $\beta$-expansions of the elements of $[\xi]$.

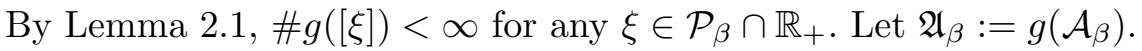


Lemma 2.2. The mapping $g: \mathcal{A}_{\beta} \rightarrow \mathfrak{A}_{\beta}$ is one-to-one.

Proof. We need to prove the injectivity of $g$ only. Let $g([\xi])=g\left(\left[\xi^{\prime}\right]\right)$. This means that there exist $\xi_{1} \in[\xi], \xi_{2} \in\left[\xi^{\prime}\right]$ whose tails of the $\beta$-expansions coincide. But this implies $\xi_{1}-\xi_{2} \in \mathbb{Z}[\beta]$, whence $[\xi]=\left[\xi^{\prime}\right]$.

Thus, we have defined a certain finite arithmetic group of classes of sequences that may be regarded as a realization of the Pisot group in $X_{\beta}$. The arithmetic operations on $\mathfrak{A}_{\beta}$ inherited from $\mathcal{A}_{\beta}$ will be denoted by $\oplus$ and $\ominus$ respectively. That is,

$$
[\bar{\varepsilon}] \oplus\left[\bar{\varepsilon}^{\prime}\right]:=g\left(g^{-1}([\bar{\varepsilon}])+g^{-1}\left(\left[\bar{\varepsilon}^{\prime}\right]\right)\right),
$$

and similarly for "circular" subtraction. Note also that for $[\bar{\varepsilon}] \in \mathfrak{A}_{\beta}$,

$$
g^{-1}([\bar{\varepsilon}])=\left[\sum_{j=-n}^{\infty} \varepsilon_{j} \beta^{-j}\right] \quad \text { for any } n \in \mathbb{Z}
$$

(the square brackets on the right-hand side denote the equivalence class in $\mathcal{A}_{\beta}$ ).

Definition. We define the natural arithmetic on $\mathfrak{A}_{\beta}$ as follows: $\bar{\varepsilon}+\bar{\varepsilon}^{\prime}$ is by definition, the class of all sequences $\bar{\varepsilon}^{\prime \prime} \in X_{\beta}$ which can be obtained as weak limits of the sequence $\left\{\varepsilon^{(N)}+\varepsilon^{\prime(N)}\right\}_{N \geq 1}$, where $\varepsilon^{(N)}=(\ldots, 0,0, \ldots, 0$, $\left.\varepsilon_{-N}, \varepsilon_{-N+1}, \ldots\right)$. The difference $\bar{\varepsilon}-\bar{\varepsilon}^{\prime}$ is defined in a similar way, namely, if $\sum_{j=-N}^{\infty}\left(\varepsilon_{j}-\varepsilon_{j}^{\prime}\right) \beta^{-j}<0$, then we take $\left(\ldots, 0,0, \ldots, 0,1, \varepsilon_{-N}, \varepsilon_{-N+1}, \ldots\right)$ instead of $\varepsilon^{(N)}$.

Proposition 2.3. The operations + and $\oplus$ (respectively - and $\ominus)$ on $\mathfrak{A}_{\beta}$ coincide.

Proof. Let $[\bar{\varepsilon}],\left[\bar{\varepsilon}^{\prime}\right] \in \mathfrak{A}_{\beta}$ and $S_{1}:=[\bar{\varepsilon}]+\left[\bar{\varepsilon}^{\prime}\right], S_{2}:=[\bar{\varepsilon}] \oplus\left[\bar{\varepsilon}^{\prime}\right]$. We first show that $S_{1} \subset S_{2}$. Let $\bar{\varepsilon}^{\prime \prime} \in[\bar{\varepsilon}]+\left[\bar{\varepsilon}^{\prime}\right]$. This means by definition that there exists a subsequence $\left\{N_{k}\right\}_{k=1}^{\infty}$ such that $\bar{\varepsilon}^{\prime \prime}=\lim _{k}\left(\varepsilon^{\left(N_{k}\right)}+\varepsilon^{\left(N_{k}\right)}\right)$. We need to show that $g^{-1}\left(\left[\bar{\varepsilon}^{\prime \prime}\right]\right)=g^{-1}([\bar{\varepsilon}])+g^{-1}\left(\left[\bar{\varepsilon}^{\prime}\right]\right)$, which follows from (2.2) with $n=N_{k}$ with $k$ large enough to ensure that $\varepsilon^{\left(N_{k}\right)}+\varepsilon^{\prime\left(N_{k}\right)}$ will coincide with $\bar{\varepsilon}^{\prime \prime}$ at, say, all positive indices.

To prove the reverse inclusion, let $\bar{\varepsilon}^{\prime \prime} \in S_{2}$. To show that $\bar{\varepsilon}^{\prime \prime} \in S_{1}$, we put $N_{k}=k r$, where $r$ is the length of a common period of $\bar{\varepsilon}, \bar{\varepsilon}^{\prime}$ and $\bar{\varepsilon}^{\prime \prime}$. By definition,

$$
\eta:=\left|\sum_{j=0}^{\infty} \varepsilon_{j}^{\prime \prime} \beta^{-j}-\sum_{j=0}^{\infty}\left(\varepsilon_{j}+\varepsilon_{j}^{\prime}\right) \beta^{-j}\right| \in \mathbb{Z}[\beta],
$$

whence

$$
\left|\sum_{j=-N_{k}}^{\infty} \varepsilon_{j}^{\prime \prime} \beta^{-j}-\sum_{j=-N_{k}}^{\infty}\left(\varepsilon_{j}+\varepsilon_{j}^{\prime}\right) \beta^{-j}\right|=\beta^{k r} \eta,
$$


and by our assumption that $\beta$ is finitary, $\eta$ has a finite $\beta$-expansion, whence by (2.3) and the definition of convergence in the weak topology,

$$
\bar{\varepsilon}^{\prime \prime}=\lim _{k}\left(\varepsilon^{\left(N_{k}\right)}+\varepsilon^{\prime\left(N_{k}\right)}\right) .
$$

The proof for subtraction is the same.

Thus, $\mathfrak{A}_{\beta}$ is an additive group of classes of sequences in $X_{\beta}$ in the sense of its natural arithmetic. Its obvious property is that it is shift-invariant, i.e. contains any sequence together with all its shifts, because addition commutes with the shift - recall that the shift of a sequence finite to the left is just the multiplication of its value by $\beta$. Pursuing the idea from Section 1 (see Lemma 1.6), we will give another way of obtaining the sequences from $\mathfrak{A}_{\beta}$, which in a sense looks more traditional (see [FrSa1] for the case of the golden mean).

Lemma 2.4. For any element $[\bar{\varepsilon}] \in \mathfrak{A}_{\beta}$ there exists an eventually nonnegative sequence $\left\{T_{n}\right\}_{n=1}^{\infty} \in R_{\beta}$ such that the set of partial limits for the sequence of its $\beta$-expansions $\left\{\bar{\varepsilon}_{n}\right\}_{n=1}^{\infty}$ is exactly the class $[\bar{\varepsilon}]$.

Proof. Take $[\xi]=g^{-1}([\bar{\varepsilon}])$, and, as above, define $T_{n}$ as the integer closest to $\xi^{\prime} \beta^{n}$ for some nonnegative $\xi^{\prime} \sim \xi$ (for example, take $\xi^{\prime}$ equal to the fractional part of $\xi$ ). Then $T_{n}=\xi^{\prime} \beta^{n}+o(1)$, and the $T_{n}$ are eventually nonnegative, as $\xi^{\prime}>0$. Hence taking the set of partial limits of its $\beta$-expansions is the same as "pulling out" the periodic tail for a $\xi \in \mathcal{P}_{\beta} \cap \mathbb{R}_{+}$, because $T_{n}-\xi^{\prime} \beta^{n} \rightarrow 0, n \rightarrow+\infty$.

ExAmples. 1. For the quadratic Pisot units $\beta^{2}=k \beta \pm 1$ we proved in Section 1 that $\mathcal{P}_{\beta}=(1 / \sqrt{D}) \cdot \mathbb{Z}[\beta]$. Since $1 / \sqrt{D}=k \beta^{2} /\left(\beta^{4}-1\right)$, all sequences in $\mathfrak{A}_{\beta}$ are of period 4,2 or 1 . Let us consider some subcases. We will use the convention to write just the period instead of the whole periodic sequence.

1.1. $\beta^{2}=\beta+1$. Here $\mathfrak{A}_{\beta}=\{0000,1000,0100,0010,0001\}$. Recall that by definition, $X_{\beta}$ does not contain sequences ending with $101010 \ldots$; that is why there is no need to identify 0000 with 1010 and 0101 . Thus, every class in $\mathfrak{A}_{\beta}$ consists of just one element. If $F_{1}=1, F_{2}=2, \ldots$ is the Fibonacci sequence, then by Lemma 2.4, the set of partial limits for the $\beta$-expansions of $\left(F_{n}\right)_{n=1}^{\infty}$ in $X_{\beta}$ will give us just the four nonzero sequences in $\mathfrak{A}_{\beta}\left(^{1}\right)$. However, this may be checked directly as well:

$$
F_{k}= \begin{cases}\beta^{k-1}+\beta^{k-5}+\beta^{k-9}+\ldots+\beta^{-k+3}+\beta^{-k}, & k \text { even, } \\ \beta^{k-1}+\beta^{k-5}+\beta^{k-9}+\ldots+\beta^{-k+5}+\beta^{-k+1}, & k \text { odd }\end{cases}
$$

$\left({ }^{1}\right)$ It is easy to see that the Fibonacci sequence is the basis of the module $R_{\beta}$, i.e. for any sequence $\left\{T_{n}\right\} \in R_{\beta}, T_{n}=\sum_{k=0}^{s} \varepsilon_{k} F_{n+k}, \varepsilon_{k} \in \mathbb{Z}, n \geq n_{0}$. 
whence the limits of $\left(F_{4 k+j}\right)_{k=1}^{\infty}$ in $X_{\beta}$ do exist for $j=0,1,2,3$ and yield the four nonzero sequences in $\mathfrak{A}_{\beta}$. For more details see [FrSa1], [SV1].

1.2. $\beta^{2}=2 \beta+1$. Here $\# \mathfrak{A}_{\beta}=8$, and $\mathfrak{A}_{\beta}=\{0000,1010,0101,2000,0200$, $0020,0002,1111\}$. The elements 1010, 0101, 1111 are of order 2, whereas all other nonzero elements are of order 4 . A similar pattern holds for $\beta^{2}=$ $k \beta+1, k$ even.

REMARK. In [FrSa2] the authors study two similar groups for arbitrary quadratic Pisot units. One of them, $H_{\beta}$, is the group of all sequences of period 4 ; its order is $k^{2} D$, and it is in fact isomorphic to $\frac{\mathbb{Z}[\beta]}{k \sqrt{D}} / \mathbb{Z}[\beta]$. Another one, $G_{\beta}$, is, on the contrary, smaller than $\mathfrak{A}_{\beta}$; it is related to a certain finite automaton. For example, in the case $\beta^{2}=2 \beta+1, G_{\beta}=$ $\{0000,0101,1010,1111\} \cong(\mathbb{Z} / 2 \mathbb{Z}) \times(\mathbb{Z} / 2 \mathbb{Z})$. In the general quadratic case one can show that $G_{\beta} \subset \mathfrak{A}_{\beta} \subset H_{\beta}$, both inclusions being, generally speaking, proper. However, in the case of the golden mean (and only in this case), $G_{\beta}=\mathfrak{A}_{\beta}=H_{\beta} \cong \mathbb{Z} / 5 \mathbb{Z}$.

2. For the tribonacci number the situation with the periods of the sequences in $\mathfrak{A}_{\beta}$ appears to be more complicated. Expanding the elements of $\mathcal{P}_{\beta}$, we see that these periods are $1,2,3$ and 10 . More precisely, besides $0, \mathfrak{A}_{\beta}$ consists of exactly 40 sequences of period 10, namely: $\{1000110000$, 1010000110,1001011000,1001101100 $\}$ together with all their shifts, two sequences of period two: $\{01,10\}$ and one sequence of period 3: 100. One may ask: where are all its shifts, shouldn't they belong to $\mathfrak{A}_{\beta}$ as well? The answer is simple: arithmetically they are all equivalent, because

$$
\left(\beta+\beta^{-2}+\beta^{-5}+\ldots\right)-\left(1+\beta^{-3}+\beta^{-6}+\ldots\right)=\frac{\beta-1}{1-\beta^{-3}}=1 \in \mathbb{Z}[\beta] .
$$

This example shows that even for a finitary $\beta$ not necessarily any $\xi \in \mathcal{P}_{\beta}$ and $\xi+l, l \in \mathbb{Z}[\beta]$ will have one and the same tail. Thus, the definition of $\mathfrak{A}_{\beta}$ as a quotient set is essential.

3. $\beta^{3}=\beta+1$. Here $D=-23$. Let us prove a simple claim about the structure of the group $\mathfrak{A}_{\beta}$ in the case of prime $|D|$.

Lemma 2.5. If $|D|$ is a prime number, then $|D|$ consists of $|D|-1$ sequences of period $|D|-1$ which are shifts of each other, and 0 .

Proof. Since $\mathfrak{A}_{\beta}$ is shift-invariant and cyclic, any $\bar{\varepsilon} \in \mathfrak{A}_{\beta} \backslash\{0\}$ belongs to it together with all its shifts, and they are arithmetically nonequivalent.

Returning to the example, the period of $\xi_{0}$ is 10000100000000100000000 , and $\mathfrak{A}_{\beta}$ consists of this sequence together with its 21 shifts and 0 .

3. Symbolic dynamics associated with the Pisot group. In this section we are going to show that a certain natural arithmetic coding of the 
companion toral automorphism is not one-to-one a.e. and that its "kernel" in fact coincides with the group $\mathfrak{A}_{\beta}$. We still assume $\beta$ to be a finitary Pisot unit.

We need to recall some basic notions and facts from hyperbolic dynamics. Let $T$ be a hyperbolic automorphism of the torus $\mathbb{T}^{m}=\mathbb{R}^{m} / \mathbb{Z}^{m}, L_{\mathrm{s}}$ and $L_{\mathrm{u}}$ denote respectively the leaves of the stable and unstable foliations passing through $\mathbf{0}$. Recall that a point homoclinic to $\mathbf{0}$ (or simply a homoclinic point) is a point belonging to $L_{\mathrm{s}} \cap L_{\mathrm{u}}$. In other words, $\mathbf{t}$ is homoclinic iff $T^{n} \mathbf{t} \rightarrow \mathbf{0}$ as $n \rightarrow \pm \infty$. The homoclinic points are a group under addition, isomorphic to $\mathbb{Z}^{m}$, and we will denote it by $\mathcal{H}(T)$. Each homoclinic point $\mathbf{t}$ can be obtained as follows: take some $\mathbf{n} \in \mathbb{Z}^{m}$ and project it onto $L_{\mathrm{u}}$ along $L_{\mathrm{s}}$ and then onto $\mathbb{T}^{m}$ by taking the fractional parts of all coordinates of the vector (see $[$ Ver] $)$.

Let $T=T(\beta)$ be the group automorphism of $\mathbb{T}^{m}$ given by the companion matrix, i.e. by

$$
M=M(\beta)=\left(\begin{array}{cccccc}
k_{1} & k_{2} & k_{3} & \ldots & k_{m-1} & k_{m} \\
1 & 0 & 0 & \ldots & 0 & 0 \\
0 & 1 & 0 & \ldots & 0 & 0 \\
\ldots & \ldots & \ldots & \ldots & \ldots & \ldots \\
0 & 0 & 0 & \ldots & 1 & 0
\end{array}\right)
$$

Then $\left(1, \beta^{-1}, \ldots, \beta^{-m+1}\right)$ is an eigenvector corresponding to the eigenvalue $\beta$.

Lemma 3.1. There exists a one-to-one correspondence between the homoclinic points and the elements of $\mathcal{P}_{\beta}$. Namely, $\mathbf{t} \in \mathcal{H}(T)$ if and only if $\mathbf{t}=\left(\xi, \xi \beta^{-1}, \ldots, \xi \beta^{-m+1}\right) \bmod \mathbb{Z}^{m}$ for some $\xi \in \mathcal{P}_{\beta}$.

Proof. Since $\beta$ is a Pisot number, $L_{\mathrm{u}}$ is one-dimensional. Hence by Vershik's theorem cited above, any homoclinic point for $T$ must be of the form $\mathbf{t}=\left(\xi, \xi \beta^{-1}, \ldots, \xi \beta^{-m+1}\right) \bmod \mathbb{Z}^{m}$. To show that $\xi$ belongs to $\mathcal{P}_{\beta}$, we observe that $T^{n} \mathbf{t}=\left(\xi \beta^{n}, \xi \beta^{n-1}, \ldots, \xi \beta^{n-m+1}\right) \bmod \mathbb{Z}^{m}$. Since $T^{n} \mathbf{t}$ must tend to $\mathbf{0}$ as $n \rightarrow \pm \infty$, we are done. The converse is obvious.

Consider first two special homoclinic points. Let $\xi_{0}$ denote the generator of the Pisot group defined in Proposition 1.4, and $\mathbf{t}_{0}$ the fundamental homoclinic point given by the formula

$$
\mathbf{t}_{0}=\left(\xi_{0}, \xi_{0} \beta^{-1}, \ldots, \xi_{0} \beta^{-m+1}\right)
$$

(we omit the natural projection of $\mathbb{R}^{m}$ onto the torus, i.e. write in the coordinates of $\mathbb{R}^{m}$ ), and

$$
\mathbf{t}_{1}:=\left(1, \beta^{-1}, \ldots, \beta^{-m+1}\right) .
$$


Consider the following two mappings from $X_{\beta}$ onto $\mathbb{T}^{m}$ :

$$
\begin{aligned}
& \varphi_{0}(\varepsilon)=\sum_{k \in \mathbb{Z}} \varepsilon_{k} T^{-k} \mathbf{t}_{0}=\lim _{N \rightarrow+\infty}\left(\sum_{k=-N}^{\infty} \varepsilon_{k} \beta^{-k}\right)\left(\begin{array}{c}
\xi_{0} \\
\xi_{0} \beta^{-1} \\
\vdots \\
\xi_{0} \beta^{-m+1}
\end{array}\right) \bmod \mathbb{Z}^{m}, \\
& \varphi_{1}(\varepsilon)=\sum_{k \in \mathbb{Z}} \varepsilon_{k} T^{-k} \mathbf{t}_{1}=\lim _{N \rightarrow+\infty}\left(\sum_{k=-N}^{\infty} \varepsilon_{k} \beta^{-k}\right)\left(\begin{array}{c}
1 \\
\beta^{-1} \\
\vdots \\
\beta^{-m+1}
\end{array}\right) \bmod \mathbb{Z}^{m} .
\end{aligned}
$$

Both mappings are well defined since $\left\|\beta^{N}\right\| \rightarrow 0$ and $\left\|\xi_{0} \beta^{N}\right\| \rightarrow 0$ as $N \rightarrow+\infty$ at an exponential rate. They are obviously continuous, and their important property is that they are bounded-to-one (see [Sch] for $\varphi_{0}$ and [Sid] for the general case of mappings of the form (4.1) - see below).

Their main value is that they both semiconjugate the shift $\tau_{\beta}$ on the compactum $X_{\beta}$ and the automorphism $T$, i.e., $\varphi_{0} \tau_{\beta}=T \varphi_{0}, \varphi_{1} \tau_{\beta}=T \varphi_{1}$. Thus, both mappings may be regarded as arithmetic codings of $T$ in the sense of [SV2], [Sid]. The mapping $\varphi_{0}$ was introduced in [SV2] for $m=2$, and in $[\mathrm{Sch}]$ for higher dimensions. At the same time, the mapping $\varphi_{1}$ had been historically the first attempt of arithmetic coding of an automorphism of the torus [Ber].

Theorem (K. Schmidt [Sch]). The mapping $\varphi_{0}$ is one-to-one on the set of doubly transitive sequences of $X_{\beta}$, i.e. on the sequences $\bar{\varepsilon}$ such that the sets $\left\{\tau_{\beta}^{n} \bar{\varepsilon}: n \geq k\right\}$ and $\left\{\tau_{\beta}^{n} \bar{\varepsilon}: n \leq-k\right\}$ are both dense in $X_{\beta}$ for every $k \geq 0$. Therefore, $\varphi_{0}$ is bijective almost everywhere with respect to the measure of maximal entropy for $\tau_{\beta}\left({ }^{2}\right)$.

REMARK. Recently the author proved that $\varphi_{0}$ is one-to-one a.e. for a wider class of Pisot units, namely, for those which we have called weakly finitary. More precisely, a Pisot unit $\beta$ is called weakly finitary if for any $x \in \mathbb{Z}[\beta]$ and any $\delta>0$ there exists $f \in \operatorname{Fin}(\beta) \cap(0, \delta)$ such that $x+f \in$ $\operatorname{Fin}(\beta)$ as well. There is a conjecture (shared by most experts in this area) that every Pisot unit is weakly finitary. For more details see [Sid].

Our goal in this section is to show that $\varphi_{1}$ is $|D|$-to-one a.e. and that $\varphi_{1}^{-1}(\mathbf{0})$, after some natural identification, will coincide with $\mathfrak{A}_{\beta}$.

Definition. We will say that $\bar{\varepsilon} \in X_{\beta}$ is equivalent to $\bar{\varepsilon}^{\prime} \in X_{\beta}$ if $\varphi_{0}(\bar{\varepsilon})=$ $\varphi_{0}\left(\bar{\varepsilon}^{\prime}\right)$.

$\left({ }^{2}\right)$ The nature of this measure is not important; in fact, one may take any shiftinvariant measure that is strictly positive on all cylinders $\left[\varepsilon_{1}=i_{1}, \ldots, \varepsilon_{k}=i_{k}\right] \subset X_{\beta}$. 
We will denote this equivalence relation by $\sim$. Let $X_{\beta}^{\prime}=X_{\beta} / \sim$; by Schmidt's theorem, $\#[\bar{\varepsilon}]=1$ for a.e. sequence $\bar{\varepsilon}$. Let addition (subtraction) in $X_{\beta}^{\prime}$ be defined via the torus, i.e., $[\bar{\varepsilon}] \pm\left[\bar{\varepsilon}^{\prime}\right]:=\varphi_{0}^{-1}\left(\varphi_{0}(\bar{\varepsilon}) \pm \varphi_{0}\left(\bar{\varepsilon}^{\prime}\right)\right)$. Obviously, $X_{\beta}^{\prime}$ is a group under addition, isomorphic to $\mathbb{T}^{m}$.

Lemma 3.2. The equivalence relation for $\mathfrak{A}_{\beta}$, described in the previous section, is precisely the restriction of the equivalence relation to $X_{\beta}$.

Proof. We need to show that if $[\bar{\varepsilon}]=\left[\bar{\varepsilon}^{\prime}\right]$ in the sense of $\mathfrak{A}_{\beta}$, then $\varphi_{0}(\bar{\varepsilon})=$ $\varphi_{0}\left(\bar{\varepsilon}^{\prime}\right)$. Similarly to the proof of Proposition 2.3, let $r \in \mathbb{N}$ be a common period of the $\beta$-expansions of $\bar{\varepsilon}$ and $\bar{\varepsilon}^{\prime}$ and $N_{k}:=k r$. Then

$$
\left|\sum_{j=-N_{k}}^{\infty}\left(\varepsilon_{j}-\varepsilon_{j}^{\prime}\right) \beta^{-j}\right|=\beta^{k r} \eta, \quad \text { where } \quad \eta=\left|\sum_{j=0}^{\infty}\left(\varepsilon_{j}-\varepsilon_{j}^{\prime}\right) \beta^{-j}\right| \in \mathbb{Z}[\beta] .
$$

Hence for $l=0,1, \ldots, m-1$,

$$
\left\|\sum_{j=-N_{k}}^{\infty}\left(\varepsilon_{j}-\varepsilon_{j}^{\prime}\right) \beta^{-j} \xi_{0} \beta^{-l}\right\| \rightarrow 0 \quad \text { as } k \rightarrow+\infty,
$$

which implies $\varphi_{0}(\bar{\varepsilon})=\varphi_{0}\left(\bar{\varepsilon}^{\prime}\right)$.

THEOREM 3.3. (i) The mapping $\varphi_{1}$ is well defined on the quotient compactum $X_{\beta}^{\prime}$.

(ii) $\varphi_{1}: X_{\beta}^{\prime} \rightarrow \mathbb{T}^{m}$ is a group homomorphism with the kernel $\mathfrak{A}_{\beta}$.

Proof. (i) We need to show that if $\varphi_{0}(\bar{\varepsilon})=\varphi_{0}\left(\bar{\varepsilon}^{\prime}\right)$, then $\varphi_{1}(\bar{\varepsilon})=\varphi_{1}\left(\bar{\varepsilon}^{\prime}\right)$. By the definition of $\varphi_{0}$, we have $\left\|\xi_{0} \beta^{j} u_{N}\right\| \rightarrow 0$ for any $j \in \mathbb{Z}$, where $u_{N}=$ $\sum_{k=-N}^{\infty}\left(\varepsilon_{k}-\varepsilon_{k}^{\prime}\right) \beta^{-k}$. By Proposition $1.2, \xi_{0}^{-1} \in \mathbb{Z}[\beta]$, whence $\left\|\beta^{j} u_{N}\right\| \rightarrow 0$ as well. Hence by the definition of $\varphi_{1}$,

$$
\left\|\varphi_{1}\left(\varepsilon^{(N)}\right)-\varphi_{1}\left(\varepsilon^{\prime(N)}\right)\right\| \leq \text { const } \cdot\left\|u_{N}\right\| \rightarrow 0
$$

(ii) As was mentioned above, the mapping $\varphi_{1}$ is bounded-to-one. Since the set $\mathcal{O}=\varphi_{1}^{-1}(\mathbf{0})$ is finite and shift-invariant, it should consist of purely periodic sequences only. Let $\bar{\varepsilon} \in \mathcal{O}$ have period $r$ and $\alpha=\sum_{k=1}^{\infty} \varepsilon_{k} \beta^{-k}$. Then by the definition of $\varphi_{1},\left\|\alpha \beta^{r N}\right\| \rightarrow 0$ as $N \rightarrow+\infty$. Considering the sequences $\tau_{\beta} \bar{\varepsilon}, \tau_{\beta}^{2} \bar{\varepsilon}, \ldots, \tau_{\beta}^{r-1} \bar{\varepsilon}$, we see that $\left\|\alpha \beta^{r N+j}\right\| \rightarrow 0$ for $j=0,1, \ldots, r-1$, whence $\left\|\alpha \beta^{N}\right\| \rightarrow 0$ as $N \rightarrow+\infty$, i.e., $\alpha \in \mathcal{P}_{\beta}$, and the claim follows from Lemma 2.2. Conversely, if $\alpha \in \mathcal{P}_{\beta}$, then $\left\|\alpha \beta^{N}\right\| \rightarrow 0$, whence $\varphi_{1}(\bar{\varepsilon})=\mathbf{0}$.

Corollary 3.4. The mapping $\varphi_{1}$ is $|D|$-to-one a.e.

Let $A=\varphi_{1} \varphi_{0}^{-1}$. This mapping is well defined a.e. on the torus, and we extend it to the whole $\mathbb{T}^{m}$ by continuity. The following claim is straightforward.

Lemma 3.5. The mapping $A: \mathbb{T}^{m} \rightarrow \mathbb{T}^{m}$ is an endomorphism of the torus. Its determinant equals $\pm D$. 
In practice, to find $A$, one might expand $\xi_{0}^{-1}$ into the sum $\sum_{k=0}^{m-1} a_{k} \beta^{k}$ with $a_{k} \in \mathbb{Z}$. Then $A=\sum_{k=0}^{m-1} a_{k} T^{k}$.

EXAMPLES. 1. $\beta^{2}=k \beta \pm 1$. Here, as we know, $\xi_{0}=1 / \sqrt{D}, \xi_{0}^{-1}=\sqrt{D}=$ $2 \beta-k$. Hence

$$
A=2 T-k I=\left(\begin{array}{cc}
2 k & \pm 2 \\
2 & 0
\end{array}\right)-\left(\begin{array}{cc}
k & 0 \\
0 & k
\end{array}\right)=\left(\begin{array}{cc}
k & \pm 2 \\
2 & -k
\end{array}\right),
$$

and $\operatorname{det} A=-D(\beta)$.

2. $\beta^{3}=\beta^{2}+\beta+1$. Here $\xi_{0}^{-1}=-1-2 \beta+3 \beta^{2}$, whence

$$
A=-I-2\left(\begin{array}{lll}
1 & 1 & 1 \\
1 & 0 & 0 \\
0 & 1 & 0
\end{array}\right)+3\left(\begin{array}{lll}
1 & 1 & 1 \\
1 & 0 & 0 \\
0 & 1 & 0
\end{array}\right)^{2}=\left(\begin{array}{ccc}
3 & 4 & 1 \\
1 & 2 & 3 \\
3 & -2 & -1
\end{array}\right),
$$

and $\operatorname{det} A=44=-D(\beta)$.

4. Generalization of the Pisot group. It is natural to ask the same sort of questions for more general arithmetic codings of $T$, i.e. for those parameterized by arbitrary homoclinic points (= by arbitrary elements of the group $\mathcal{P}_{\beta}$ ). Let $\varphi_{\xi}: X_{\beta} \rightarrow \mathbb{T}^{m}$ be defined as follows:

$$
\begin{aligned}
\varphi_{\xi}(\bar{\varepsilon}) & =\sum_{k \in \mathbb{Z}} \varepsilon_{k} T^{-k} \mathbf{t} \\
& =\lim _{N \rightarrow+\infty}\left(\sum_{k=-N}^{\infty} \varepsilon_{k} \beta^{-k}\right)\left(\begin{array}{c}
\xi \\
\xi \beta^{-1} \\
\vdots \\
\xi \beta^{-m+1}
\end{array}\right) \bmod \mathbb{Z}^{m},
\end{aligned}
$$

where $\xi=\xi(\mathbf{t}) \in \mathcal{P}_{\beta}$ (see Lemma 3.1). The questions are the following:

(1) What is the value of $\# \varphi_{\xi}^{-1}(x)$ for a typical $x \in \mathbb{T}^{m}$ ?

(2) How to describe the kernel of $\varphi_{\xi}$ ?

The next assertion answers the first question; it is a generalization of the corresponding result proven in [SV2] for $m=2$.

TheOREm 4.1. For a.e. $x \in \mathbb{T}^{m}$ with respect to the Haar measure,

$$
\# \varphi_{\xi}^{-1}(x) \equiv|D N(\xi)|
$$

where $N(\cdot)$ denotes the norm of an element of the extension $\mathbb{Q}(\beta) / \mathbb{Q}$.

Proof. Let $\ell:=\xi / \xi_{0} \in \mathbb{Z}[\beta]$. If $\ell=\sum_{i=0}^{m-1} c_{i} \beta^{i}$, then

$$
\varphi_{\xi}=A_{\xi} \varphi_{0}
$$

where $A_{\xi}$ is the endomorphism of $\mathbb{T}^{m}$ given by $A_{\xi}=\sum_{i=0}^{m-1} c_{i} T^{i}$. Indeed, for the basis sequence $e^{(0)}=(\ldots, 0,0, \ldots, 0,1,0, \ldots, 0,0, \ldots)$ with the unity at the first coordinate, we have 


$$
\begin{aligned}
\left(A_{\xi} \varphi_{0}\right)\left(e^{(0)}\right) & =A_{\xi}\left(\xi_{0}, \xi_{0} \beta^{-1}, \ldots, \xi_{0} \beta^{-m+1}\right) \bmod \mathbb{Z}^{m} \\
& =\sum_{i=0}^{m-1} c_{i} T^{i}\left(\xi_{0}, \xi_{0} \beta^{-1}, \ldots, \xi_{0} \beta^{-m+1}\right) \bmod \mathbb{Z}^{m} \\
& =\sum_{i=0}^{m-1} c_{i} \beta^{i}\left(\xi_{0}, \xi_{0} \beta^{-1}, \ldots, \xi_{0} \beta^{-m+1}\right) \bmod \mathbb{Z}^{m} \\
& =\left(\xi, \xi \beta^{-1}, \ldots, \xi \beta^{-m+1}\right) \bmod \mathbb{Z}^{m}=\varphi_{\xi}\left(e^{(0)}\right) .
\end{aligned}
$$

Since the relation (4.2) is shift-invariant, it holds for any $\bar{\varepsilon}=\tau_{\beta}^{j}\left(e^{(0)}\right), j \in \mathbb{Z}$, and therefore for any $\bar{\varepsilon} \in X_{\beta}$ by linearity and continuity.

As $\varphi_{0}$ is one-to-one a.e., $\varphi_{\xi}$ will be $K$-to-one a.e. with $K=\left|\operatorname{det} A_{\xi}\right|$. By definition, $N(\ell)$ is the determinant of the matrix of the multiplication operator $x \mapsto \ell x$ in the standard basis of $\mathbb{Q}(\beta)$, whence $N(\ell)=\operatorname{det} A_{\xi}$, because $T$ is given by the companion matrix. Finally, $N(\ell)=N(\xi) / N\left(\xi_{0}\right)=$ $D N(\xi)$, as by the result of [Sam, Section 2.7], $N\left(\xi_{0}\right)=1 / D$ whenever $\xi_{0}$ is as in Proposition 1.2.

To answer the second question, let us recall that by definition of Section $3, X_{\beta}^{\prime}$ is the quotient group $X_{\beta} / \sim$ with respect to the equivalence relation: $\bar{\varepsilon} \sim \bar{\varepsilon}^{\prime}$ iff $\varphi_{0}(\bar{\varepsilon})=\varphi_{0}\left(\bar{\varepsilon}^{\prime}\right)$.

Proposition 4.2. The kernel of the mapping $\varphi_{\xi}$ is a finite subgroup of $X_{\beta}^{\prime}$ whose size is $|D N(\xi)|$. Any element $\bar{\varepsilon} \in \operatorname{Ker} \varphi_{\xi}$ can be obtained as follows: take some $\varkappa \in \mathbb{Z}[\beta]$ such that $\alpha=\left(\xi_{0} / \xi\right) \varkappa>0$ and let $\bar{\varepsilon}$ be the purely periodic sequence whose period coincides with the one of the $\beta$-expansion of $\alpha$.

Proof. The first claim is a direct consequence of Theorem 4.1. To prove the second one, suppose $\varphi_{\xi}(\bar{\varepsilon})=\mathbf{0}$. By the same reasons as above, $\bar{\varepsilon}$ must be purely periodic. Let $\alpha=\sum_{k=1}^{\infty} \varepsilon_{k} \beta^{-k}$. By (4.1), $\left\|\xi \alpha \beta^{n}\right\| \rightarrow 0$ as $n \rightarrow+\infty$, whence by Proposition $1.2, \xi \alpha=\xi_{0} \varkappa, \varkappa \in \mathbb{Z}[\beta]$. Hence $\alpha=\left(\xi_{0} / \xi\right) \varkappa$.

Conversely, if $\bar{\varepsilon}$ is the two-sided continuation of the period of $\varkappa \xi_{0} / \xi>0$ for some $\varkappa \in \mathbb{Z}[\beta]$, and $\alpha=\sum_{k=1}^{\infty} \varepsilon_{k} \beta^{-k}$, then $\left\|\alpha \xi \beta^{n}\right\|=\left\|\xi_{0} \varkappa \beta^{n}\right\|+o(1) \rightarrow$ 0 as $n \rightarrow+\infty$, whence by formula $(4.1), \varphi_{\xi}(\bar{\varepsilon})=\mathbf{0}$.

REMARK. In practice, to obtain $\operatorname{Ker} \varphi_{\xi}$, one may take $\varkappa=1, \beta, \ldots, \beta^{m-1}$ and consider the linear span of the corresponding set of sequences.

Corollary 4.3. For any finitary Pisot $\beta$ and any purely periodic sequence $\bar{\varepsilon} \in X_{\beta}$ there exists $\xi \in \mathcal{P}_{\beta}$ such that $\bar{\varepsilon} \in \operatorname{Ker} \varphi_{\xi}$.

Proof. Let $r$ denote the period of $\bar{\varepsilon}$. One may take $\xi=\left(\beta^{r}-1\right) \xi_{0}$ and apply (4.1).

ExAmple. Let $\beta=(1+\sqrt{5}) / 2$. Here, as we know, $\xi_{0}=1 / \sqrt{5}$. Note first that if $\ell=\xi / \xi_{0}$ is not a unit of the $\operatorname{ring} \mathbb{Z}[\beta]$ (i.e., it is not of the 
form $\pm \beta^{k}$ for some $k \in \mathbb{Z}$ ), then, as is well known, $|N(\ell)| \geq 4$, whence by Theorem 4.1, the number of pre-images for $\varphi_{\xi}$ is greater than or equal to 4 as well. This corresponds to the observation that the smallest period of a nonzero sequence in $X_{\beta}$ is three (as $\overline{01} \notin X_{\beta}$ ). Therefore, the set $\mathfrak{A}=$ $\{0,100,010,001\}$ is the smallest possible (in capacity) shift-invariant subset containing 0 and something else. As $1 / 2=\beta^{-2}+\beta^{-5}+\beta^{-8}+\ldots$, by Proposition $4.2, \mathfrak{A}$ is indeed the kernel of the mapping $\varphi_{\xi}$ with $\xi=2 / \sqrt{5}$. Obviously, in this case $A_{\xi}=\left(\begin{array}{ll}2 & 0 \\ 0 & 2\end{array}\right)$.

Thus, Ker $\varphi_{2 / \sqrt{5}}$ consists of all the sequences of period 3 and is in fact isomorphic to $(\mathbb{Z} / 2 \mathbb{Z}) \times(\mathbb{Z} / 2 \mathbb{Z})$. It is curious that $\operatorname{Ker} \varphi_{4 / \sqrt{5}}$ is just the set of all sequences of period 6 ; it is isomorphic to $(\mathbb{Z} / 2 \mathbb{Z}) \times(\mathbb{Z} / 2 \mathbb{Z}) \times$ $(\mathbb{Z} / 4 \mathbb{Z})$.

Acknowledgements. I wish to thank Anatoly Vershik for our fruitful collaboration in dimension two that has given rise to some ideas in the present paper for higher dimensions. An essential part of this work was written in June-July 1999 during my stay in LIAFA at University Paris-VII, and I am indebted to Christiane Frougny for the invitation and stimulating discussions. I am grateful to Shigeki Akiyama for his professional numbertheoretic advice and his active interest in this paper.

\section{References}

[Ak1] S. Akiyama, Cubic Pisot units with finite beta-expansions, in: Algebraic Number Theory and Diophantine Analysis (Graz, 1998), de Gruyter, Berlin, 2000, 11-26.

[Ak2] - On the boundary of self-affine tilings generated by Pisot numbers, J. Math. Soc. Japan, to appear.

[Ber] A. Bertrand-Mathis, Développement en base $\theta$, répartition modulo un de la suite $\left(x \theta^{n}\right)_{n \geq 0}$; langages codés et $\theta$-shift, Bull. Soc. Math. France 114 (1986), 271-323.

[Bl] F. Blanchard, $\beta$-expansions and symbolic dynamics, Theoret. Comput. Sci. 65 (1989), 131-141.

[Cas] J. W. S. Cassels, An Introduction in Diophantine Approximation, Cambridge Univ. Press, 1957.

[DuPi] J. Dufresnoy et C. Pisot, Sur un ensemble fermé d'entiers algébriques, Ann. Sci. École Norm. Sup. (3) 70 (1953), 105-133.

[FrolTa] A. Frölich and M. Taylor, Algebraic Number Theory, Cambridge Univ. Press, 1991.

[FrSa1] C. Frougny and J. Sakarovitch, Automatic conversion from Fibonacci representation to representation in base $\varphi$, and a generalization, Internat. J. Algebra Comput. 9 (1999), 351-384.

[FrSa2] -,-, Two groups associated with quadratic Pisot units, submitted.

[FrSo] C. Frougny and B. Solomyak, Finite beta-expansions, Ergodic Theory Dynam. Systems 12 (1992), 713-723. 
[Pa] W. Parry, On the $\beta$-expansions of real numbers, Acta Math. Acad. Sci. Hungar. 11 (1960), 401-416.

[Sam] P. Samuel, Algebraic Theory of Numbers, Houghton Mifflin, Boston, 1970.

[Sch] K. Schmidt, Algebraic codings of expansive group automorphisms and two-sided beta-shifts, Monatsh. Math. 129 (2000), 37-61.

[Sid] N. Sidorov, Bijective and general arithmetic codings for Pisot toral automorphisms, J. Dynam. Control Systems, to appear.

[SV1] N. Sidorov and A. Vershik, Ergodic properties of Erdös measure, the entropy of the goldenshift, and related problems, Monatsh. Math. 126 (1998), 215-261.

[SV2] - - Bijective arithmetic codings of hyperbolic automorphisms of the 2-torus, and binary quadratic forms, J. Dynam. Control Systems 4 (1998), 365-400.

[Ver] A. M. Vershik, Arithmetic isomorphism of the toral hyperbolic automorphisms and sofic systems, Functional Anal. Appl. 26 (1992), 170-173.

Department of Mathematics

UMIST

P.O. Box 88

Manchester M60 1QD, United Kingdom

E-mail: Nikita.A.Sidorov@umist.ac.uk

Received on 13.1.2000

and in revised form on 11.6.2001 